\title{
Quantitative modeling of the temperature-dependent internal quantum efficiency in InGaN light emitting diodes
}

\author{
Anna Nirschl ${ }^{*, 1,2}$, Alvaro Gomez-Iglesias', Matthias Sabathil', Georg Hartung', Jürgen Off', \\ and Dominique Bougeard ${ }^{2}$ \\ ${ }^{1}$ OSRAM Opto Semiconductors GmbH, Leibnizstraße 4, 93055 Regensburg, Germany \\ ${ }^{2}$ Institut für Experimentelle und Angewandte Physik, Universität Regensburg, Universitätsstraße 31, 93040 Regensburg, Germany
}

Received 11 March 2014, revised 26 May 2014, accepted 11 June 2014

Published online 7 July 2014

Keywords GaN, InGaN, internal quantum efficiency, light emitting diodes

*Corresponding author: e-mail anna.nirschl@osram-os.com, Phone: +49 151 46758077, Fax: +49 9418501090

The temperature dependence of the internal quantum efficiency (IQE) of blue InGaN-based light emitting diodes is analyzed both experimentally and theoretically with a drift-diffusion transport model. A high-performance reference structure and two improved epitaxial designs are compared at different operating temperatures. In contrast to a simple $\mathrm{ABC}$ model, the proposed approach allows for quantitative predictions of IQEs including optimizations regarding spatial carrier distributions at room temperature. At elevated temperatures, a moderate increase of the Auger coefficient gives a more precise agreement between experiment and simulations. The results show that the model is suitable to quantitatively predict the IQE for different structures and temperatures.
1 Introduction InGaN-based LEDs suffer from a drop of the internal quantum efficiency (IQE) at current densities higher than $1-10 \mathrm{~A} \mathrm{~cm}^{-2}$. This so called droop is one of the most controversially discussed topics in the field of III-nitride semiconductors. Possible contributions to this effect proposed in the literature are carrier leakage [1-4], non-radiative recombination via defects [5-7], polarization fields [8], and Auger recombination [9-15]. A popular tool for empirical IQE predictions is the so called $A B C$ rate equation model $[9,12]$, which maps the influence of several mechanisms in a first approximation, e.g., the dependence of the recombination coefficients on band gap energy [16, 17]. The drawback of this model is that it does not include local information about carriers, thus it is not able to predict recent IQE improvements obtained by homogenization of carrier density in the active region [18, 19]. Furthermore, the applicability of the model at realistic application temperatures is rarely addressed.

In this letter, IQE measurements are analyzed in combination with simulations to understand the role of the spatial distribution of the charge carriers in the LED performance as a function of current and temperature. We extend the $\mathrm{ABC}$ model by introducing locally resolved carriers through self-consistent band structure and transport calculations of the active region. In addition, we discuss the temperature dependence of the empirical model. A good agreement between experiment and theory is obtained for different epitaxial structures with a single consistent set of material parameters, justifying the modeling approach taken.

2 Experiment and model All samples discussed in this letter were grown by metal-organic vapor phase epitaxy on (0001) sapphire substrates. They share a common epitaxial structure comprising a low-temperature $\mathrm{GaN}$ nucleation layer, followed by a thick n-doped $\mathrm{GaN}$ buffer, an active region consisting of five InGaN quantum wells (QWs) embedded in GaN barriers, a p-doped AlGaN electron blocking layer, and a final p-doped GaN cap layer.

The epitaxial structures were processed into $1 \mathrm{~mm}^{2}$ thinfilm flip-chips [20] and temperature dependent electrooptical characterization was performed on unpackaged devices. Several nominally identical chips were measured. A low deviation of all structures from the target peak emission at $439 \mathrm{~nm}$ was observed.

The IQE is defined as the fraction of the total applied current $(I)$, which recombines radiatively in the active 
region $\left(I_{\text {rad }}\right)$

$$
\mathrm{IQE}=\frac{I_{\mathrm{rad}}}{I}
$$

and was determined from the light output $\left(\Phi_{\mathrm{e}}\right)$, taking the extraction effciency $(\mathrm{EXE}=86 \%$ [12]) from fitted raytracing simulations

$$
\Phi_{\mathrm{e}}=I \cdot \mathrm{IQE} \cdot E_{\mathrm{ph}} \cdot \mathrm{EXE}
$$

where $E_{\mathrm{ph}}$ is the photon energy. All experimentally extracted IQE values at a given current density are averaged over all characterized chips in order to minimize potential errors.

Typically, IQE measurements are fitted with a simple $\mathrm{ABC}$ rate equation model [9, 12], which is based on rough approximations: the electron and hole concentrations are not only assumed to be equal but also constant in the whole recombination volume. As a result, this model provides some insight into the LED material parameters but cannot deliver quantitative predictions. For example, since the charge carrier distribution is very inhomogeneous in AlInGaN multi-quantum well structures due to the interface polarization charges and the low hole mobility, IQE improvements due to a better carrier concentration profile cannot be reproduced. Taking into account the spatial distribution of electrons and holes is therefore essential. Thus, to circumvent these limitations, we have carried out carrier transport simulations coupled to a spatially resolved ABC model. The generalized Shockley-Read-Hall (SRH), radiative and Auger recombination rates in one dimension are given by

$$
\begin{aligned}
& R_{\mathrm{SRH}}(x)=\frac{n(x) \cdot p(x)-n_{\mathrm{i}}^{2}(x)}{\tau_{\mathrm{p}} \cdot\left[n(x)+n_{\mathrm{T}}(x)\right]+\tau_{\mathrm{n}} \cdot\left[p(x)+p_{\mathrm{T}}(x)\right]} \\
& \begin{aligned}
& R_{\mathrm{rad}}(x)=B \cdot\left[n(x) \cdot p(x)-n_{\mathrm{i}}^{2}(x)\right] \\
& R_{\text {Auger }}(x)= C_{\mathrm{p}} \cdot n(x) \cdot\left[p^{2}(x)-n_{\mathrm{i}}^{2}(x)\right] \\
&+C_{\mathrm{n}} \cdot p(x) \cdot\left[n^{2}(x)-n_{\mathrm{i}}^{2}(x)\right]
\end{aligned}
\end{aligned}
$$

where $n_{\mathrm{i}}$ is the intrinsic carrier concentration and $\tau_{\mathrm{n}}$ and $\tau_{\mathrm{p}}$ are the SRH recombination lifetimes for electrons and holes, respectively. $n_{\mathrm{T}}$ and $p_{\mathrm{T}}$ are the electron and hole concentrations if the Fermi level coincides with the energy level of the non-radiative recombination center. In the active region we assume deep traps, which lie approximately in the middle of the band gap [21, 22]. Hence, these densities can be simplified to $n_{\mathrm{T}}=p_{\mathrm{T}}=n=n_{\mathrm{i}} . B$ is the bimolecular recombination parameter and $C_{\mathrm{n}}$ and $C_{\mathrm{p}}$ are the Auger coefficients for electron-electron-hole and hole-holeelectron processes, respectively. In this work, $C=C_{\mathrm{n}}=C_{\mathrm{p}}$ has been assumed for simplicity. At last, $n(x)$ and $p(x)$ are the position-dependent electron and hole densities along the growth direction.
These recombination rates are coupled to one-dimensional Schrödinger-Poisson and drift-diffusion equations [23, 24], which were solved self-consistently using the software package nextnano ++ . Finally, the IQE is given by

$$
\mathrm{IQE}=\eta_{\text {inj }} \cdot \frac{\int R_{\mathrm{rad}}(x) \mathrm{d} x}{\int R_{\mathrm{SRH}}(x)+R_{\mathrm{rad}}(x)+R_{\text {Auger }}(x) \mathrm{d} x},
$$

where each of the recombination rates is integrated over the active volume and $\eta_{\text {inj }}$ represents the injection efficiency. The IQE measurement at room temperature of a reference structure, presented below, was fitted first to obtain the $\tau=\tau_{\mathrm{n}}=\tau_{\mathrm{p}}=3 \times 10^{-7} \mathrm{~s}, B=1.4 \times 10^{-11} \mathrm{~cm}^{3} \mathrm{~s}^{-1}$, and $C=6 \times 10^{-31} \mathrm{~cm}^{6} \mathrm{~s}^{-1}$ parameters. The values were found to be in the range of those recently reported by different groups $[11,13,16]$. Most importantly, the same ABC and band parameters (see, e.g., Vurgaftman and Meyer [25]) were used to model all epitaxial structures.

3 Spatially resolved $A B C$ model Three structures were investigated. The reference is a high-performance LED test structure. Two more structures, named type 1 and type 2 in the following, were designed to induce a more uniform carrier distribution in the active region compared to the reference. Both design variations follow the approach of Wang et al. [18] and Zhang et al. [19] where IQE improvements at room temperature were observed when improving the uniformity of the carrier distribution by gradually varying the quantum well thicknesses. In our samples, we slightly reduced the indium content of one quantum well to the next from the $\mathrm{n}$-side toward the $\mathrm{p}$-side. This leads to a gradually increasing band gap across the multi-quantum well region, which engineers the local carrier distribution as described by Zhang et al. [19]: The holes can more easily tunnel into the neighboring quantum well on the $\mathrm{n}$-side due to the lower ground state energy level. On the other hand, the electron transport coming from the deepest quantum state on the n-side is impeded. This difference in indium content and thus step in energy level from one quantum well to the next is higher in type 2 than in type 1 . Figure 1 displays the simulation of the electron and hole distributions in the active region of the three structures. As intended, type 2 exhibits the most uniform carrier distribution.

Figure 2 shows the experimentally (symbols) determined and simulated (solid lines) IQEs at room temperature as a function of the current density. The typical droop behavior is experimentally observed for all three structures and indeed correlates with the inhomogeneity of the carrier distribution in the active region. The simulations based on Eq. (6) are in excellent quantitative agreement with the experimental data of all three structures. These results demonstrate that, by taking into account the distribution of electrons and holes, our spatially resolved $\mathrm{ABC}$ model is able to quantitatively predict design variations in the active region of the LEDs at room temperature. None of the tested structures shows carrier overflow, leading to an injection 

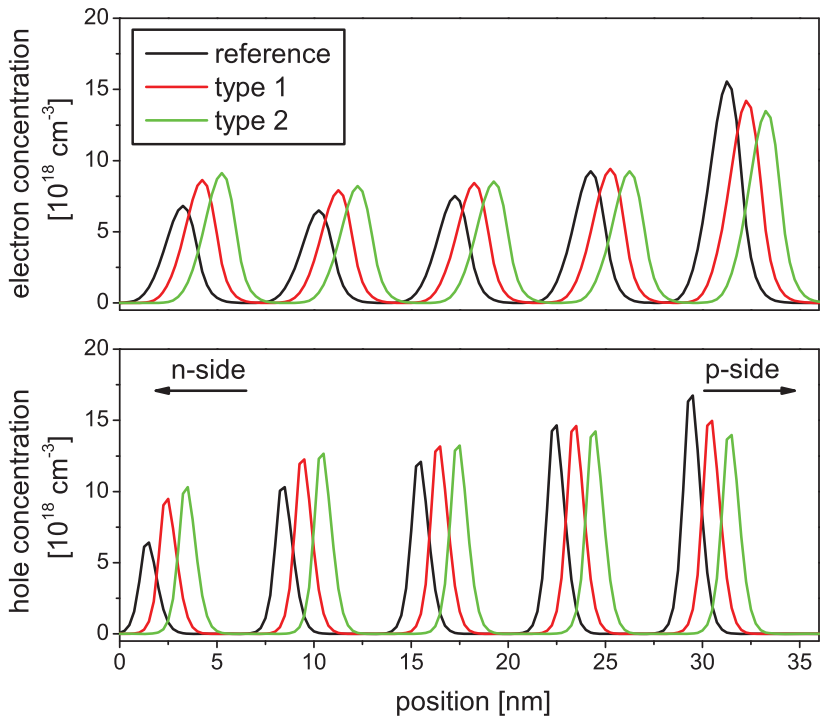

Figure 1 Carrier concentrations at $50 \mathrm{Acm}^{-2}$ and room temperature.

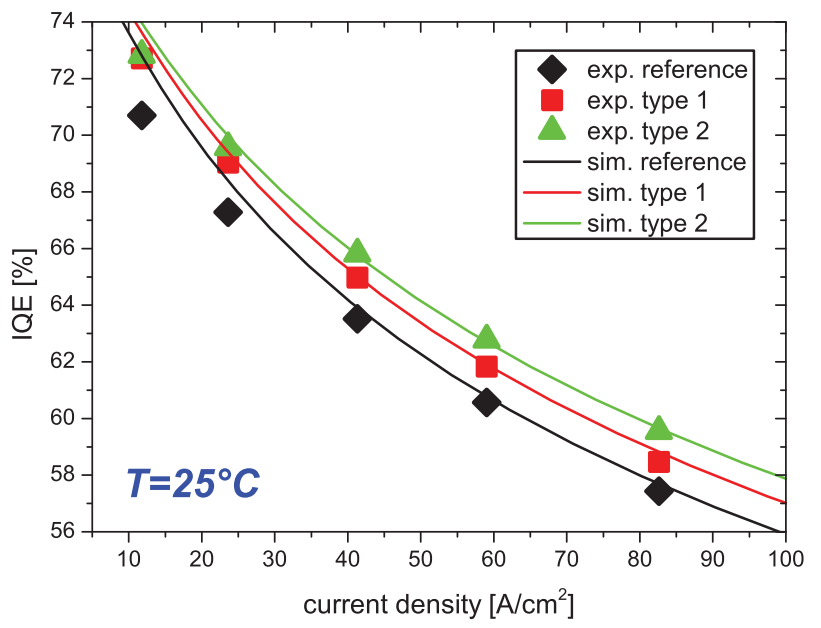

Figure 2 Comparison of IQEs determined from measurements (symbols) and simulations using a space-resolved ABC-model combined with self-consistent Schrödinger-Poisson and driftdiffusion calculations (solid lines) at room temperature.

efficiency equal to unity. Since SRH recombination is negligible in these high current regimes, Eq. (6) then implies that the IQE is dominated by the Auger-like recombination term. Given the fact that the Auger recombination varies with the cube of the carrier density, it can then be understood that significant improvements of the IQE can be obtained when optimizing the homogeneity of the carrier distribution in the active region, as observed for the structures type 1 and 2 compared to the reference structure at room temperature.

4 Temperature dependence To check the suitability of the model for realistic applications, the temperaturedependent performance of the three structures was studied.

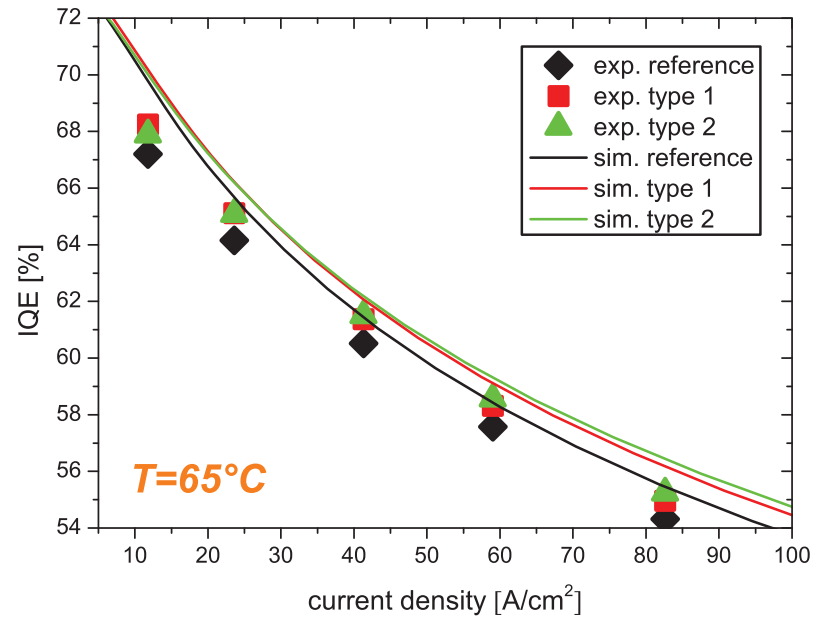

Figure 3 Comparison of IQEs determined from measurements (symbols) and simulations using a space-resolved ABC-model combined with self-consistent Schrödinger-Poisson and driftdiffusion calculations (solid lines) at $65^{\circ} \mathrm{C}$.

For simulations at higher temperatures, the following assumptions were made: first, the SRH recombination rate increases with temperature as described by two activation energies $E_{1}=163.7 \mathrm{meV}$ and $E_{2}=20.8 \mathrm{meV}$, which were determined from previous experiments using a similar approach to that described by Galler et al. [16]. The origin of these energies can be explained by the barrier heights for thermal delocalization of excitons from potential fluctuations and diffusion to dislocations [26]. Furthermore, a $B$ coefficient inversely proportional to temperature is included to follow theoretical expectations for a two-dimensional system [27]. In a first instance, the Auger coefficient was set to be temperature independent as reported previously [12]. In Fig. 3, the IQE extracted from a measurement at $65^{\circ} \mathrm{C}$ is shown and compared to simulations. The IQE is reduced for all structures compared to the results at $25^{\circ} \mathrm{C}$. Although the carrier transport improves at elevated temperatures due to the increasing occupation probability at higher energies, the reduction of the radiative coefficient dominates. Furthermore, the effects of design variations on the IQE, which were obvious at $25^{\circ} \mathrm{C}$ disappear with increasing temperature. Thermal energy enhances the carrier transport also in the reference, thus closing the efficiency gap between the considered structures. The model is able to predict the redistribution of carriers with temperature and thus describes the decreasing advantage in IQE due to heating of the junction.

As can be seen from Fig. 3, the absolute values of the simulated IQE do not agree as well with the experiment as at room temperature. The deviations become even more pronounced at $85^{\circ} \mathrm{C}$ where all three epitaxial structures show almost the same IQE, which does not coincide with the simulation result of the reference overlaying the calculation values of type 1 and type 2 . This indicates that the assumption of a $T$-independent Auger coefficient may not be 


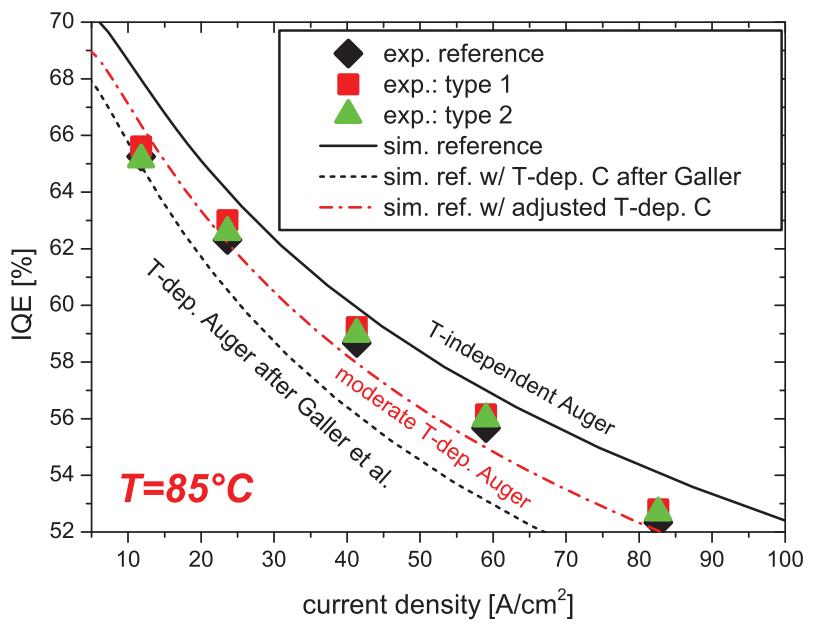

Figure $4 \mathrm{IQE}$ at $85^{\circ} \mathrm{C}$ determined from measurements of the three structures (symbols) compared to calculations of the reference, which overlays the simulations for type 1 and type 2, using an Auger coefficient identical to the one at room temperature (solid line), which is temperature-activated based on results published by Galler et al. [16] (dashed line) and with a moderate temperature dependence according to Eq. (7) (dash-dotted line).

appropriate well above room temperature. Unfortunately, due to the lack of consensus on the physical origin of Auger recombination in InGaN and the complexity of calculations for QWs [28-30], discordant theoretical results of Auger coefficients exist [31]. Hence, simulations were carried out, including an empirical temperature dependence based on data published by Galler et al. [16]. This temperature dependence shows a too strong reduction in IQE as can be seen from the dashed line in Fig. 4.

The fact that the trend reported by Galler et al. was obtained by averaging over all investigated samples with peak wavelengths ranging from 428 to $457 \mathrm{~nm}$ may explain the discrepancy, because the thermal enhancement of the Auger coefficient depends on the band gap [11,32] and also on the confinement [28, 33]. We suggest a thermal enhancement of the Auger coefficient slightly weaker than the one from Galler et al. A temperature dependence of

$$
C(T)=0.5 \cdot\left(\frac{T}{300 \mathrm{~K}} \cdot C(300 \mathrm{~K})+C(300 \mathrm{~K})\right)
$$

delivers a good approximation of the IQE as can be seen from the dash-dotted line in Fig. 4. It should be mentioned, that also the recalculated results for $65^{\circ} \mathrm{C}$ show better agreement with the experiment. Further refinement of the formula could be achieved by taking into account a distinction between the type of Auger recombination since the work carried out by Galler et al. [34] shows that the coefficient for electron-electron-hole is higher than for electron-hole-hole Auger processes.
5 Conclusions In summary, temperature-dependent IQEs were determined from measurements of LEDs with different active regions and compared to simulations. A spatially resolved $\mathrm{ABC}$ model in combination with Schrödinger-Poisson and drift-diffusion calculations has only once been calibrated and then quantitatively reproduced the experimental results of all structures at room temperature. At higher junction temperatures an Auger-coefficient with moderate temperature dependence was taken into account. For all structures and temperatures no carrier overflow was observed, thus the only origin of the droop in the simulations is the Auger-like recombination mechanism. The suggested model is able to predict improvements of the $\mathrm{IQE}$ at room temperature resulting from more uniform carrier distributions in optimized multi-quantum well structures. Moreover, this work showed that the closing of efficiency gap experimentally observed at higher temperatures can be simulated and attributed to the thermally enhanced carrier transport.

\section{References}

[1] M. H. Kim, M. F. Schubert, Q. Dai, J. K. Kim, E. F. Schubert, J. Piprek, and Y. Park, Appl. Phys. Lett. 91, 183507 (2007).

[2] S. Steiger, R. Veprek, and B. Witzigmann, J. Comput. Electron. 7, 509 (2008).

[3] X. Ni, X. Li, J. Lee, S. Liu, V. Avrutin, U. Özgür, H. Morkoç, A. Matulionis, T. Paskova, G. Mulholland, and K. R. Evans, Phys. Status Solidi RRL 4, 194 (2010).

[4] D. S. Meyaard, G. B. Lin, J. Cho, E. F. Schubert, H. Shim, S. H. Han, M. H. Kim, C. Sone, and Y. S. Kim, Appl. Phys. Lett. 102, 251114 (2013).

[5] T. Mukai, M. Yamada, and S. Nakamura, Jpn. J. Appl. Phys. 38, 3976 (1999).

[6] A. Hangleiter, C. Netzel, D. Fuhrmann, F. Hitzel, L. Hoffmann, H. Bremers, U. Rossow, G. Ade, and P. Hinze, Philos. Mag. 87, 2041 (2007).

[7] B. Monemar and B. E. Sernelius, Appl. Phys. Lett. 91, 181103 (2007).

[8] L. Wang, C. Lu, J. Lu, L. Liu, N. Liu, Y. Chen, Y. Zhang, E. Gu, and X. Hu, Opt. Express 19, 14182 (2011).

[9] Y. C. Shen, G. O. Mueller, S. Watanabe, N. F. Gardner, A. Munkholm, and M. R. Krames, Appl. Phys. Lett. 91, 141101 (2007).

[10] J. Hader, J. V. Moloney, B. Pasenow, S. W. Koch, M. Sabathil, N. Linder, and S. Lutgen, Appl. Phys. Lett. 92, 261103 (2008).

[11] E. Kioupakis, P. Rinke, K. T. Delaney, and C. G. Van de Walle, Appl. Phys. Lett. 98, 161107 (2011).

[12] A. Laubsch, M. Sabathil, J. Baur, M. Peter, and B. Hahn, IEEE Trans. Electron Devices 57, 79 (2010).

[13] M. Brendel, A. Kruse, H. Jönen, L. Hoffmann, H. Bremers, U. Rossow, and A. Hangleiter, Appl. Phys. Lett. 99, 031106 (2011).

[14] J. Iveland, L. Martinelli, J. Peretti, J. S. Speck, and C. Weisbuch, Phys. Rev. Lett. 110, 177406 (2013).

[15] M. Binder, A. Nirschl, R. Zeisel, T. Hager, H. J. Lugauer, M. Sabathil, D. Bougeard, J. Wagner, and B. Galler, Appl. Phys. Lett. 103, 071108 (2013). 
[16] B. Galler, P. Drechsel, R. Monnard, P. Rode, P. Stauss, S. Froehlich, W. Bergbauer, M. Binder, M. Sabathil, B. Hahn, and J. Wagner, Appl. Phys. Lett. 101, 131111 (2012).

[17] D. Schiavon, M. Binder, M. Peter, B. Galler, P. Drechsel, and F. Scholz, Phys. Status Solidi B 250, 283 (2013).

[18] C. H. Wang, S. P. Chang, W. T. Chang, J. C. Li, Y. S. Lu, Z. Y. Li, H. C. Yang, H. C. Kuo, T. C. Lu, and S. C. Wang, Appl. Phys. Lett. 97, 181101 (2010).

[19] J. Y. Zhang, L. E. Cai, B. P. Zhang, X. L. Hu, F. Jiang, J. Z. Yu, and Q. M. Wang, Appl. Phys. Lett. 95, 161110 (2009).

[20] V. Haerle, B. Hahn, S. Kaiser, A. Weimar, S. Bader, F. Eberhard, A. Plössl, and D. Eisert, Phys. Status Solidi A 201, 2736 (2004).

[21] M. A. Reshchikov and H. Morkoç, J. Appl. Phys. 97, 061301 (2005).

[22] A. Armstrong, T. A. Henry, D. D. Koleske, M. H. Crawford, and S. R. Lee, Opt. Express 20, A812 (2012).

[23] A. Trellakis, T. Zibold, T. Andlauer, S. Birner, R. Smith, R. Morschl, and P. Vogl, J. Comput. Electron. 5, 285 (2006).

[24] S. Birner, S. Hackenbuchner, M. Sabathil, G. Zandler, J. A. Majewski, T. Andlauer, T. Zibold, R. Morschl, A. Trellakis, and P. Vogl, Acta Phys. Polon. A 110, 111 (2006).
[25] I. Vurgaftman and J. R. Meyer, J. Appl. Phys. 94, 3675 (2003).

[26] J. Abell and T. D. Moustakas, Appl. Phys. Lett. 92, 091901 (2008).

[27] E. Rosencher and B. Vinter, Optoelectronics (Cambridge University Press, Cambridge, 2002).

[28] F. Bertazzi, X. Zhou, M. Goano, G. Ghione, and E. Bellotti, Appl. Phys. Lett. 103, 081106 (2013).

[29] E. Kioupakis, Q. Yan, and C. G. Van de Walle, Appl. Phys. Lett. 101, 231107 (2012).

[30] R. Vaxenburg, A. Rodina, E. Lifshitz, and A. L. Efros, Appl. Phys. Lett. 103, 221111 (2013).

[31] E. Kioupakis, Q. Yan, D. Steiauf, and C. G. Van de Walle, New J. Phys. 15, 125006 (2013).

[32] F. Bertazzi, M. Goano, and E. Bellotti, Appl. Phys. Lett. 101, 011111 (2012).

[33] A. S. Polkovnikov and G. G. Zegrya, Phys. Rev. B 58, 4039 (1998).

[34] B. Galler, H. J. Lugauer, M. Binder, R. Hollweck, Y. Folwill, A. Nirschl, A. Gomez-Iglesias, B. Hahn, J. Wagner, and M. Sabathil, Appl. Phys. Express 6, 112101 (2013). 